\title{
II peer-assessment nella formazione insegnanti: accorgimenti e ricadute
}

\author{
Peer-assessment and teachers' training: Tips and impacts
} Nadia Sansone*, Ilaria Bortolotti ${ }^{* *}$, Manuela Fabbri ${ }^{* \star *}$

\section{Riassunto}

Il contributo indaga la competenza valutativa di insegnanti in servizio coinvolti in un'attività di peer-assessment e le loro percezioni circa gli impatti della pratica in sé. Il contesto dello studio è rappresentato da un Master universitario di I livello basato sull'Approccio Trialogico all'Apprendimento in cui i partecipanti sperimentano metodologie didattiche innovative, dalla progettazione all'implementazione alla valutazione. Dopo aver descritto nel dettaglio modalità e procedure delle attività di peer-assessment oggetto dello studio, si illustra il metodo di analisi quali-quantitativa dei dati raccolti: 407 rubrics compilate da 43 insegnanti e 28 questionari semi-strutturati a supporto della riflessione finale attorno all'attività.

Nel complesso, le analisi mostrano come l'attività di peer-assessment così come proposta abbia stimolato, da un lato, il potenziamento di specifiche competenze professionalizzanti, dall'altro, la motivazione ad apprendere e il sentimento di appartenenza a una comunità di pratiche. Vengono discussi gli elementi a supporto dell'efficacia della pratica valutativa e le possibili ricadute in aula.

Parole chiave: peer-assessment, rubrics, formazione insegnanti, feedback, Approccio Trialogico all'Apprendimento, riflessione sulle pratiche

\section{Abstract}

The article investigates the evaluative competence of in-service teachers involved in a peer-assessment activity and their perceptions about the impacts of the practice itself. The context of the study is represented by a Level I

\footnotetext{
${ }^{*}$ Ricercatrice in Pedagogia sperimentale, Dipartimento di Scienze Giuridiche ed Economiche, Unitelma Sapienza, Università di Roma, E-mail: nadia.sansone@unitelmasapienza.it.

** Assegnista di Ricerca, Dipartimento di Psicologia dei processi di Sviluppo e Socializzazione, Università Sapienza di Roma, E-mail: ilaria.bortolotti@uniroma1.it.

${ }^{* * *}$ Professoressa Associata in Didattica generale e Pedagogia speciale, Università di Bologna, E-mail: m.fabbri@unibo.it.

Il presente contributo è frutto del lavoro congiunto delle tre autrici. Tuttavia, sono attribuibili a: Nadia Sansone il paragrafo 2 e 4; a Ilaria Bortolotti il paragrafo 3; a Manuela Fabbri i paragrafi 1 e 5 .
}

Doi: $10.3280 /$ ess2-2021oa12481 
University Master based on the Trialogical Learning Approach in which participants experiment with innovative teaching methodologies, from design to implementation to evaluation. After having described in detail the methods and procedures of the peer-assessment activities covered by the study, the method of the quali-quantitative analysis of the collected data is illustrated: 407 rubrics compiled by 43 teachers and 28 semi-structured questionnaires to support the final reflection around the activities.

Overall, the analyzes show how the peer-assessment activity just as proposed has stimulated, on the one hand, the enhancement of specific professional skills, on the other, the motivation to learn and the feeling of belonging to a community of practices. The elements supporting the effectiveness of the evaluation practice and the possible repercussions in the classroom are discussed.

Keywords: peer-assessment, rubrics, teachers' training, feedback, Trialogical Learning Approach, reflecting on practices

Articolo sottomesso: 07/09/2021, Accettato: 06/10/2021

Disponibile online: 21/12/2021

\section{Cornice teorica}

1.1 Dall'educational evaluation all'assessment for learning: il ruolo del feedback nel peer-assessment

Negli ultimi decenni la letteratura scientifica sull'educational evaluation ha spostato gradualmente la sua attenzione da un approccio prettamente sommativo, eccessivamente focalizzato sulla mera certificazione degli apprendimenti conseguiti dagli studenti, ad uno più qualitativo, aprendosi a dimensioni maggiormente formative e virando dunque verso il costrutto di assessment for learning (Black et al., 2003; Leahy et al., 2005; Wiliam et al., 2004). All'interno di questa cornice, il feedback - tradizionalmente inteso come uno strumento educativo in grado di rinforzare e migliorare le prestazioni e l'apprendimento degli studenti (Sadler, 1989; Lipnevich e Smith, 2009) -, diventa un inner generative process (Nicol, 2018) grazie al quale il discente, da un lato, riflette sul proprio processo di apprendimento, dall'altro, costruisce la conoscenza attraverso i propri atti valutativi (Grion et al., 2017). In quanto tale, il feedback rappresenta l'elemento chiave di una delle principali pratiche in ambito di assessment for learning: il peer assessment. Con questa definizione si intende il 
processo valutativo effettuato tra pari, chiamati a fornire feedback quantitativi e qualitativi sulle performance e sui prodotti di compagni o colleghi e a riceverne, supportati da momenti di discussione e riflessione (Strijbos e Sluijsmans, 2010). La valutazione tra pari si configura, infatti, come un dispositivo educativo-formativo di natura fortemente meta-riflessiva che non si riduce «alla mera attribuzione di punteggi che andrebbe a inibire, o quanto meno, distorcere le potenzialità della revisione tra pari», ma si traduce, piuttosto, in «un processo di riflessione critica che si conclude con la definizione di un feedback costruttivo che gli studenti sono invitati a offrirsi» (Cesareni e Sansone, 2019, p. 142). In questo senso, la strategia del peer assessment diventa fonte di vantaggio non solo e non tanto per chi riceve un feedback, quanto per chi valuta (Grion et al., 2017; Grion e Tino, 2018). In particolare, l'atto di valutare può essere considerato un learning tool proprio perché permette ai pari che valutano di acquisire competenza nella formulazione di giudizi, di migliorare la capacità di autoregolare il proprio apprendimento, di favorire una comprensione più profonda dell'oggetto di conoscenza (Foschi, Cecchinato e Say, 2019; Topping, 1998).

\subsection{Formare i docenti al peer-assessment}

La ricerca scientifica attuale appare consapevole della necessità di riflettere sia sul cambiamento del concetto di valutazione, sia sul legame esistente tra la validità della valutazione tra pari e gli accorgimenti di progettazione e implementazione (Topping, 2017; 2021). L'efficacia delle pratiche di peer-assessment $\mathrm{e}$, in generale, la promozione di una literacy valutativa all'interno delle istituzioni scolastiche ed universitarie appare, infatti, necessariamente legata alla sua collocazione all'interno di una ragionata progettazione didattica (Carless et al., 2017). Quando, infatti, si coinvolgono gli studenti in attività di peer assessment è importante curare i dettagli pratico-organizzativi e attenuare possibili criticità: dall'inefficacia e scarsa obiettività della pratica in assenza di una guida chiara da parte del docente, ai vissuti di disagio e riluttanza degli studenti nel formulare giudizi critico-negativi verso i pari (Hanrahan e Isaacs, 2001; Rushton, Ramsey e Rada, 1993). Risultano dunque centrali momenti di formazione e modellamento in cui esplicitare le modalità e i criteri con cui svolgere l'attività, con l'ausilio di griglie di valutazione ed esemplificazioni in grado di aumentare l'efficacia del peer-assessment, permettendo a chi formula il feedback di sperimentare alcune competenze critiche e, a chi le riceve, di trarne vantaggio (Gielen, Dochy e Dierick, 2003; Hattie e Timperley, 2007). In particolare, assumono un ruolo chiave le rubric, intese come strumenti in grado di promuovere motivazione, auto-efficacia e risultati di apprendimento (Topping, 2017). 
Nel complesso, la forza didattica di questi dispositivi valutativi, dalle griglie alle rubrics, poggia su una diffusa trasparenza di base dei criteri di valutazione che pari o docenti utilizzeranno, fino ad arrivare a forme di negoziazione condivisa. Secondo Topping (2021), infatti, l'esplicitazione e la chiarezza dei criteri di valutazione rappresenta un fattore essenziale per il successo dell'esperienza formativa, in senso lato, e della pratica valutativa, in senso stretto. Si rafforza, in questo modo, una cultura positiva della valutazione, intesa sia come accettazione del proprio limite, sia come forma di collaborazione per il miglioramento dei processi e prodotti dei pari.

All'interno di questo approccio valutativo risulta evidentemente chiave per gli insegnanti l'acquisizione di saperi scientifico-professionali specifici (Giovannini et al., 2017; Magnoler, Notti e Perla, 2017; Rivoltella e Rossi, 2017; Sibilio e Aiello, 2018; Vannini, 2019). Da qui l'urgenza di percorsi di formazione dedicati in cui poter sperimentare in prima persona metodologie di peerassessment, così da saggiare potenzialità e limiti delle stesse, fino ad individuare le modalità contestuali e personali di trasferimento di quelle pratiche nel proprio ambito operativo.

Simili percorsi di formazione richiedono l'implementazione di approcci didattici innovativi, capaci di coinvolgere i formandi in attività autentiche di costruzione di artefatti condivisi che, attraverso varie fasi di commenti reciproci e revisioni successive, favoriscano una riflessione che vada dalle pratiche alla propria professionalità (Sansone et al., 2019; Sansone e Ritella, 2020).

\section{Contesto e metodo della ricerca}

\subsection{II contest}

Il contesto di questa ricerca è dato dal Master universitario di I livello "Tecnologie per l'apprendimento e per lo sviluppo di competenze" (TASK), erogato in modalità e-Learning dall'ateneo Unitelma Sapienza di Roma. Il percorso si basa su un modello di formazione esperienziale e metodologicamente fondato attorno ai principi dell'Approccio Trialogico all'Apprendimento (Sansone et al., 2016; Sansone et al., 2020), per cui i discenti sono chiamati a realizzare insieme oggetti condivisi e significativi, attraverso la mediazione di strumenti digitali e partecipando ad attività diversificate di riflessione, revisione e miglioramento dei propri prodotti. Infatti, alla fruizione autonoma di contenuti teorici in modalità di autoapprendimento, il Master TASK affianca specifiche attività online, le etivities (Sansone, 2020) al fine di promuovere competenze professionali e, soprattutto, di offrire l'occasione di sperimentare quelle metodologie 
che gli insegnanti potranno riportare nella propria pratica didattica, avendone provato direttamente potenzialità e vincoli. Proprio nell'ambito di alcune di queste etivities sono stati raccolti e analizzati i dati qui considerati: l'etivity "Peer-assessment dei prodotti di gruppo" e l'etivity "Io, dopo il peer-assessment". Le due etivities seguono e concludono un'attività di gruppo denominata "Contest multimediale" in cui gli insegnanti formandi $(\mathrm{N}=73$, età media $=46)$ organizzati spontaneamente in 15 gruppi di quattro componenti - hanno realizzato una risorsa didattica multimediale composita costituita da video-pillola, infografica e quiz di valutazione, seguendo specifiche consegne in merito a: caratteristiche dei prodotti, modalità e tempistiche di svolgimento dell'attività, distribuzione interna dei compiti nel gruppo. Infine, a corredo delle consegne, i gruppi di lavoro hanno ricevuto precise indicazioni sui criteri di valutazione che sarebbero stati usati dai docenti del Master per commentare i prodotti, attraverso la condivisione della rubric (vedi Appendice 1) ${ }^{1}$. Una volta costruiti e condivisi i prodotti in una repository online dedicato, durante l'etivity "Peerassessment dei prodotti di gruppo", gli insegnanti hanno utilizzato la rubric per osservare, valutare e commentare i prodotti dei colleghi; in seguito, nell'etivity "Io, dopo il peer-assessment", sono stati invitati a riflettere sulla stessa, guidati da specifici stimoli.

\subsection{Obiettivi e metodo della ricerca}

L'obiettivo di questo studio è duplice:

1. da un lato, si vuole osservare la competenza valutativa in atto degli insegnanti quando sono chiamati ad integrare giudizi quantitativi e feedback qualitativi;

2. dall'altro lato, si vuole esplorare la percezione degli insegnanti circa il valore dell'attività di peer-assessment, sia ai fini del proprio sviluppo formativo e professionale, sia immaginando di proporla in aula ai propri studenti.

Per il primo obiettivo, si è proceduto ad analizzare le valutazioni espresse $(\mathrm{N}=407)$ dagli insegnanti $(\mathrm{N}=43)$ attraverso le rubric. In particolare: a. le medie dei punteggi attribuiti ai prodotti sono state confrontate con le valutazioni medie dei docenti del Master, quindi effettuati opportuni test di significatività (Test di Kruskal-Wallis a campioni indipendenti); b. i feedback qualitativi sono stati analizzati da due giudici indipendenti ${ }^{2}$, quindi calcolato l'accordo

\footnotetext{
${ }^{1}$ La rubric si compone di indicatori specifici per ogni micro-prodotto (totale 12 indicatori). Per ciascun indicatore sono previsti quattro possibili livelli e un feedback di argomentazione del livello attribuito.

${ }^{2}$ I giudici hanno osservato quanto ciascun feedback fosse chiaro, esaustivo e corredato da indicazioni precise e/o esempi. Nelle osservazioni si sono serviti di una scala Likert a 5 punti: 1 tutti i commenti sono vaghi, incompleti o poco chiari; 2 molti commenti sono vaghi, incompleti
} 
intergiudice, per ogni prodotto, attraverso il coefficiente $\mathrm{W}$ di Kendall $(\mathrm{W}=$ 0,87 per la video-pillola, $\mathrm{W}=0,94$ per l'infografica, $\mathrm{W}=0,89$ per il quiz).

Per il secondo obiettivo, al termine dell'attività di peer-assessment, agli insegnanti è stato proposto di compilare online e in forma anonima un modulo di supporto alla riflessione ( $\mathrm{N}=28)$. Il modulo (Appendice 2) è composto da tre stimoli: un primo stimolo a risposta chiusa contenente sette item che indagano le percezioni individuali sul livello di acquisizione di conoscenze e competenze professionali e metacognitive, di senso di appartenenza alla comunità di pratiche e di apprezzamento dei prodotti dei colleghi. Gli ultimi due stimoli sono rappresentati da domande aperte che mirano ad elicitare una riflessione critica rispetto a due temi centrali: 1. 1'apprendimento implicito che deriva dal confrontare il proprio lavoro con quello altrui, in termini di individuazione dei punti forti e deboli dei propri prodotti e di possibili modalità di miglioramento degli stessi (Domanda "Cambiamenti di ritorno"), 2. l'impatto dell'attività di peer-assessment rispetto alle proprie convinzioni, credenze, idee in tema di valutazione (Domanda "Scoperte"). Lo stimolo a risposta chiusa è stato analizzato attraverso statistiche descrittive, mentre gli stimoli aperti sono stati analizzati da due valutatori indipendenti che hanno applicato sistemi categoriali creati ad hoc (Appendice 3 e 4), confrontandosi fino a raggiungere un accordo totale sui casi dubbi.

\section{Risultati}

\subsection{La competenza valutativa degli insegnanti in un'attività di peer-assessment}

Le analisi dei punteggi attribuiti dagli insegnanti ai propri pari mostrano un sostanziale allineamento con i punteggi degli esperti (coefficiente di concordanza di Kendall $>.70$ ), riportando differenze statisticamente non significative (Test di Kruskal-Wallis a campioni indipendenti con $\mathrm{p}=.05$ ) (Tab. 1).

Tab. 1 - Confronto valutazioni pari ed esperti (Livelli Rubrica 1:4)

\begin{tabular}{ccccc}
\hline Prodotto & & Indicatori & \\
\hline Infografica & Rappresentatività & $\begin{array}{c}\text { Coerenza } \\
\text { interna }\end{array}$ & $\begin{array}{c}\text { Correttezza dei } \\
\text { contenuti }\end{array}$ & Stile \\
\hline
\end{tabular}

o poco chiari; 3 alcuni commenti sono vaghi, incompleti o poco chiari; 4 tutti i commenti sono chiari e specifici; 5 tutti i commenti sono chiari, specifici, corredati da esempi concreti e riccamente argomentati. 
Education Sciences \& Society, 2/2021 ISSN 2038-9442, ISSNe 2284-015X

\begin{tabular}{cccccc} 
esperti & 3,43 & 3,66 & 3,60 & 3,57 & \\
pari & 3,46 & 3,36 & 3,69 & 3,26 & \\
\hline Quiz & Rappresentatività & Struttura & $\begin{array}{c}\text { Aspetti } \\
\text { valutativi }\end{array}$ & & \\
\hline esperti & 3,52 & 3,50 & 3,48 & & \\
pari & 3,55 & 3,56 & 3,53 & & \\
\hline video-pillola & Rappresentatività & Esposizione & $\begin{array}{c}\text { Supporto } \\
\text { grafico }\end{array}$ & $\begin{array}{c}\text { Coerenza } \\
\text { narrativa }\end{array}$ & Montaggio \\
\hline esperti & 3,75 & 3,44 & 3,44 & 3,81 & 3,35 \\
pari & 3,68 & 3,58 & 3,60 & 3,76 & 3,25 \\
\hline
\end{tabular}

Osservando i punteggi specifici, si nota come sia gli insegnanti sia gli esperti attribuiscono punteggi medi superiori al livello 3 dei 4 presenti in rubrica (Tab. 1). I punteggi più alti si registrano per la coerenza narrativa della video-pillola (punteggio medio $=3,76$ ), la correttezza dei contenuti dell'infografica (punteggio medio $=3,69)$ e la rappresentatività della video-pillola (punteggio medio $=3,68)$.

Tab. 2 - Il feedback dei pari osservato rispetto al livello di concretezza (Scala Likert 1:5)

\begin{tabular}{cccccc}
\hline Prodotto & & \multicolumn{3}{c}{ Indicatori } \\
\hline & Rappresentatività & $\begin{array}{c}\text { Coerenza } \\
\text { interna }\end{array}$ & $\begin{array}{c}\text { Correttezza } \\
\text { dei contenuti }\end{array}$ & Stile & \\
\hline Infografica & 3,16 & 3,50 & 3,31 & 3,48 & \\
\hline & Rappresentatività & Struttura & $\begin{array}{c}\text { Aspetti valu- } \\
\text { tativi }\end{array}$ & & \\
\hline Quiz & 3,49 & 3,50 & 3,57 & Montaggio \\
\hline Rappresentatività & Esposizione & $\begin{array}{c}\text { Supporto } \\
\text { grafico }\end{array}$ & $\begin{array}{c}\text { Coerenza } \\
\text { narrativa }\end{array}$ & \\
\hline $\begin{array}{c}\text { video- } \\
\text { pillola }\end{array}$ & 3,85 & 3,95 & 3,44 & 4,00 & 4,03 \\
\hline
\end{tabular}


La tabella 2 ci restituisce una visione complessiva rispetto alla qualità del feedback fornito dagli insegnanti ai propri pari, osservato rispetto al livello di concretezza.

I feedback qualitativi che gli insegnanti offrono a supporto dei punteggi attribuiti per ogni indicatore risultano mediamente concreti; si tratta, cioè, di commenti sufficientemente capaci di indirizzare l'attenzione dei colleghi sugli aspetti di migliorabilità e/o di argomentare giudizi di per sé positivi, grazie alla presenza diffusa di indicazioni chiare ed esempi specifici. In particolare, la maggiore concretezza si riscontra nei feedback offerti al prodotto video-pillola con 4 indicatori su 5 (rappresentatività, esposizione, coerenza narrativa e montaggio) i cui commenti sono stati valutati con punteggi superiori a 3,85:

«Il montaggio è ben realizzato, forse molto denso e sbilanciato dal punto di vista grafico (scritte e immagini di accompagnamento) rispetto all'audio, che è limitato all'uso di musiche le quali, per quanto coerenti con quanto viene espresso, sono appunto sbilanciate. Il canale visivo è preponderante rispetto a quello uditivo. Aggiungerei una voce narrante che possa sostituire alcune informazioni veicolate attraverso immagini e/o scritte.» (Oggetto video-pillola, Feedback indicatore Montaggio).

Riteniamo utile evidenziare come la specificità e ricchezza dei commenti non sia una prerogativa dei punteggi più bassi. Spesso, infatti, è proprio in corrispondenza di punteggi massimi (livello 4 degli indicatori) che gli insegnanti hanno offerto argomentazioni ricche e complete per la valutazione attribuita:

«Da questo punto di vista l'infografica si merita un applauso, in quanto riesce a richiamare - anche grazie alla scelta vincente nella video-pillola - in modo immediato i vari passaggi. L'unico consiglio che mi sentirei di dare è di salvarla in PDF, invece che in formato immagine, e inserire dei link, dove ci sono i punti che introducono le varie sezioni che mandano al punto preciso del video dove viene trattato, in modo da favorire il ritrovamento della sezione se si desidera approfondire. In ogni caso, anche in considerazione che la video-pillola è stata caricata su Youtube, come minimo metterei un link a video nel footer dell'infografica (magari anche nel formato qrcode, in caso di fruizione dell'infografica stampata)» (Oggetto Infografica, Feedback indicatore Rappresentatività).

\subsection{La riflessione sull'attività di peer-assessment: percezioni di valore ed efficacia}

L'attività di peer-assessment viene complessivamente valutata come molto efficace dagli insegnanti che vi hanno preso parte e hanno successivamente risposto agli stimoli di riflessione forniti all'interno del percorso del Master (Fig. $1)$. 


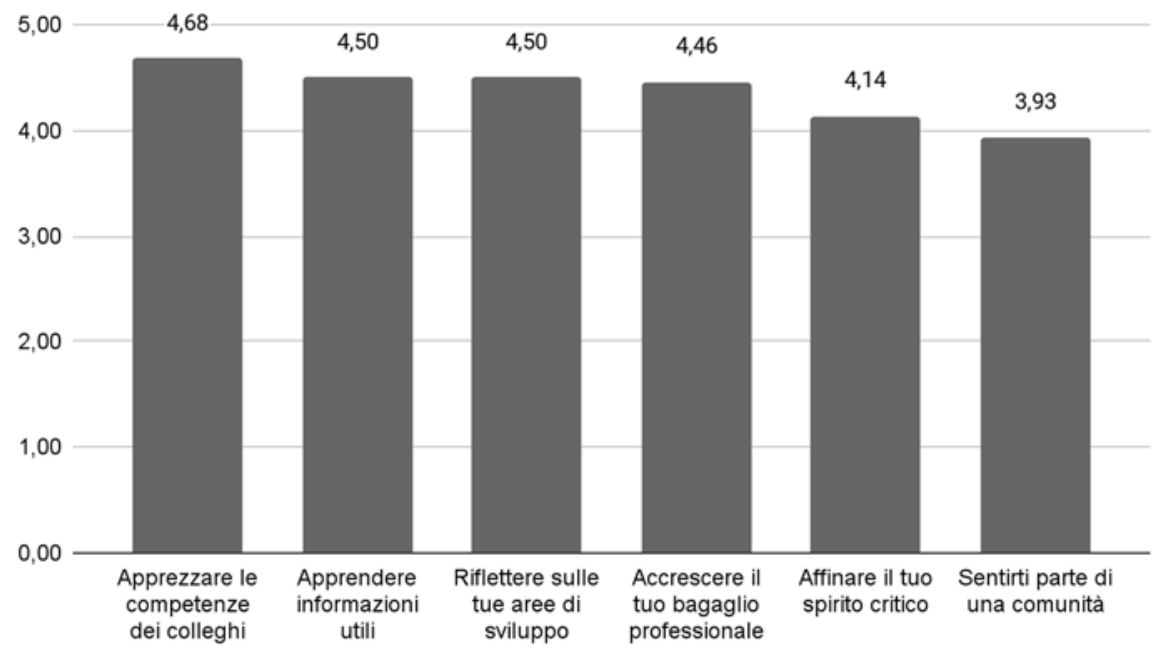

Fig. 1 - Il peer-assessment secondo gli insegnanti: efficacia ed utilità (Scala Likert 1:5)

In particolare, attraverso il peer-assessment gli insegnanti ritengono di aver potuto apprezzare maggiormente le competenze dei colleghi (media $=4,68)$, di aver appreso informazioni utili legate ai temi oggetto dei prodotti (media $=$ 4,50 ) e, di conseguenza, di aver accresciuto il proprio bagaglio professionale in relazione a strumenti e metodologie $($ media $=4,46)$. Alla possibilità di osservare i prodotti altrui è, del resto, collegata la riflessione sulle proprie aree di sviluppo (media $=4,50$ ), e l'affinamento del proprio spirito critico (media $=$ $4,14)$. Non meno importante l'impatto relazionale percepito dell'attività di peer-assessment: sentirsi parte di una comunità di pratiche, infatti, è un'altra ricaduta positiva avvertita dagli insegnanti al termine di questa attività (media $=3,93$ ).

L'analisi categoriale delle risposte aperte ha generato 47 segmenti per la domanda "Cambiamenti di ritorno" (media segmenti per rispondente: 1,7) e 39 segmenti per la domanda "Scoperte" (media segmenti per rispondente: 1,4).

Ad una prima macro-categorizzazione, la maggior parte dei cambiamenti che i rispondenti apporterebbero ai propri prodotti dopo aver visionato quelli dei colleghi riguarda aspetti narrativo-contenutistici (38\%). In molti casi (26\%), tuttavia, i rispondenti non si focalizzano sui possibili cambiamenti, ma estendono la propria riflessione, ad esempio riconoscendo la qualità del lavoro dei pari, del proprio o dell'intera attività svolta durante la realizzazione dei prodotti. Il $23 \%$ degli insegnanti si sofferma poi su aspetti tecnici, mentre il $13 \%$ ritiene non vi sia nulla da migliorare nel proprio prodotto. 
L'analisi di secondo livello dei segmenti delle risposte alla domanda "Cambiamenti di ritorno" (Fig.2) sottolinea la preponderanza dei due descrittori della categoria aspetti narrativo-contenutistici con i loro $21 \%$ (coerenza e chiarezza narrativa) e $17 \%$ (struttura interna dell'oggetto).

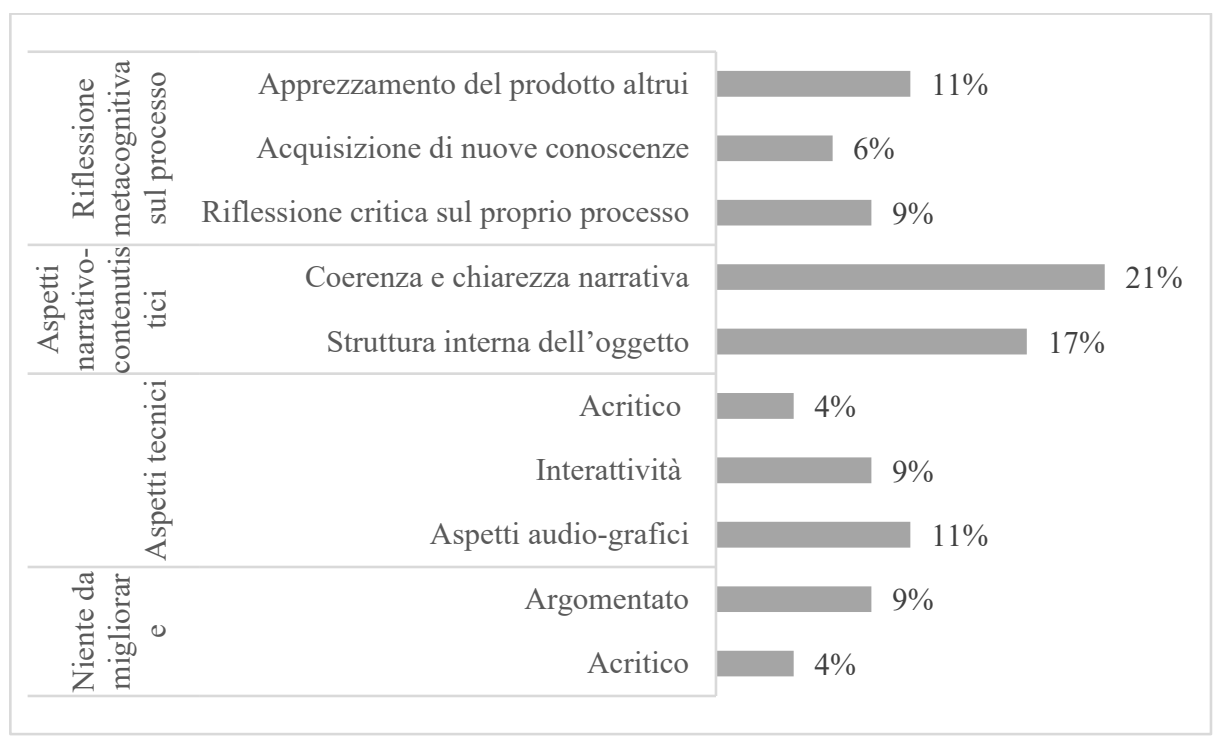

Fig. 2 - I cambiamenti di ritorno specifici

Descrittori quali "Apprezzamento del prodotto altrui" e "Aspetti audio-grafici" con il loro $11 \%$ di frequenze percentuali rappresentano l'elemento più diffuso delle rispettive categorie (Riflessione metacognitiva sul processo; Aspetti tecnici). Val la pena sottolineare come, anche laddove i rispondenti non individuano alcun aspetto da migliorare a valle del confronto coi prodotti dei colleghi, nel $9 \%$ dei casi argomentano la propria risposta: «Per la nostra infografica è stata proposta una soluzione altamente bilanciata per contenuti testuali e grafici, tali da essere riassuntivi ed esplicativi del contenuto della video-pillola, perciò non cambierei niente».

Per i rispondenti, del resto, la principale scoperta realizzata alla fine dell'attività di peer-assessment si concretizza proprio in termini di una riflessione metacognitiva (64\%) circa le pratiche valutative in genere o l'esperienza concreta appena vissuta. Seguono le scoperte relative all'acquisizione di informazioni e conoscenze (18\%) e al riconoscimento di sé stessi o dei colleghi (18\%).

Guardando al dettaglio dei descrittori (Fig. 3), il descrittore sostanzialmente più diffuso appartiene - ancora una volta - alla categoria riflessiva: l'efficacia dell'attività di peer-assessment (26\%) è la scoperta più importante per i partecipanti: «L'opportunità di aver affinato il mio spirito critico è senz'altro quello che più mi ha sorpreso alla fine di un'attività che mi ha permesso di apprendere e accrescere il mio bagaglio professionale». 
Education Sciences \& Society, 2/2021 ISSN 2038-9442, ISSNe 2284-015X

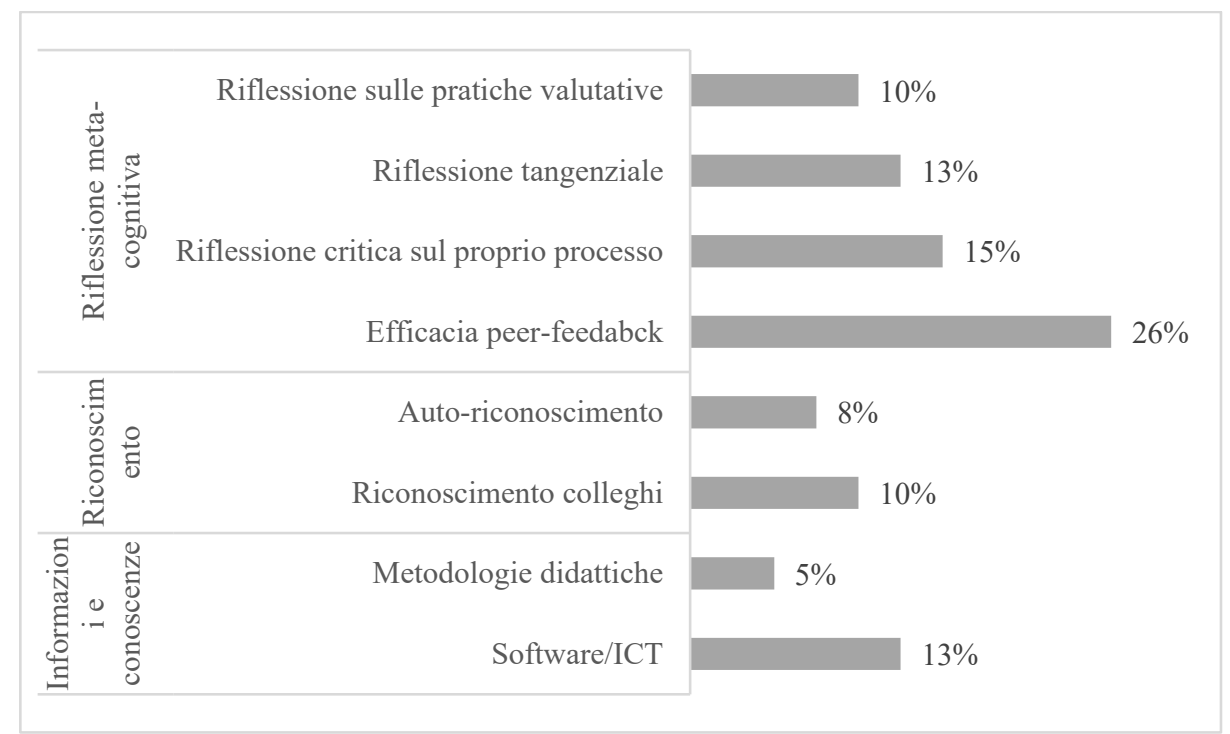

Fig. 3 - Le scoperte specifiche

Tra le scoperte di contenuto spiccano quelle legate alla possibilità di conoscere nuovi software e tool digitali grazie all'analisi dei prodotti oggetto del peer-assessment $(13 \%)$. Infine, si conferma anche in questo stimolo, la possibilità di riconoscere le competenze dei colleghi (10\%), cui si aggiunge quella di auto-riconoscersi (8\%): «Ho scoperto di essere capace in poco tempo di lavorare con prodotti mai utilizzati prima, divertendomi a scoprire le potenzialità degli strumenti»

\section{Discussione}

Il primo obiettivo di questo studio è stato quello di osservare la competenza valutativa degli insegnanti quando sono chiamati ad integrare giudizi quantitativi basati su rubric e feedback qualitativi, ovvero quando il focus del processo valutativo si sposta dalla mera certificazione degli obiettivi raggiunti alla possibilità di sostenere il percorso di apprendimento individuale, attraverso una precisa indicazione dei punti di forza e di miglioramento, altresì suggerendo possibili strategie per colmare i gap identificati.

Dall'analisi quali-quantitativa dell'attività di peer-assessment effettuata dagli insegnanti del Master TASK traspare una buona competenza valutativa, sia quando chiamati ad applicare indicatori e livelli delle rubric, sia quando chiamati a formulari feedback argomentativi a supporto dei livelli attribuiti. Nel primo caso, infatti, le valutazioni degli insegnanti formandi sono analoghe a 
quelle degli esperti di contenuto del Master. Questo primo dato può essere ricondotto alla professionalità e competenza pregressa dei partecipanti; si tratta, infatti, di insegnanti con vari anni di servizio e con un'alta inclinazione a metodologie di insegnamento e valutazione diversificate e innovative. Tuttavia, è utile ricordare che, prima dell'applicazione della rubric, gli insegnanti hanno avuto molteplici occasioni di utilizzo e riflessione sulla stessa, con ciò acquisendo una chiara comprensione su come applicare i criteri previsti e sviluppando competenze critiche via via più raffinate (Liu e Li, 2014), come riportano gli stessi partecipanti all'attività quando parlano di una «capacità critica continuamente sviluppata grazie alla definizione chiara di standard prestazionali di valutazione». La presenza di valide guide per la valutazione, del resto, è uno dei pilastri fondamentali per la realizzazione di percorsi di peer-assessment formativamente efficaci; ed è proprio in questi percorsi che le valutazioni dei formandi tendono ad essere analoghe a quelle degli esperti (Panadero, AlonsoTapia e Huertas, 2012; Orpen, 1982). Nel secondo caso, i feedback forniti dagli insegnanti risultano complessivamente di buona qualità: i commenti sono chiari e sufficientemente corredati da indicazioni specifiche, sia quando usati per argomentare l'attribuzione di bassi punteggi, sia quando integrano valutazioni alte. L'esercizio di questa competenza appare di cruciale importanza in vista di un trasferimento pratico nella propria pratica didattica quotidiana: sia che si tratti di valorizzare la buona performance di un collega o di uno studente, sia che si stia modellando il processo del dare feedback coi propri discenti, l'individuazione dei punti di forza risulta un'azione molto più complessa rispetto al tracciamento dell'errore e, allo stesso modo, sostanzialmente più capace di generare circoli virtuosi di rinforzo della motivazione ad apprendere, del senso di appartenenza e, in generale, di autostima e benessere (Schartel, 2012).

Il secondo obiettivo del nostro studio è stato esplorare la percezione degli insegnanti circa il valore dell'attività di peer-assessment, sia ai fini del proprio sviluppo formativo e professionale, sia come pratica da riportare in aula coi propri studenti. L'analisi congiunta delle risposte chiuse e aperte fornite dai rispondenti rimanda ad un generale apprezzamento, richiamato in primo luogo dalle percezioni di efficacia mediamente molto alte che i partecipanti attribuiscono all'attività, in termini di impatti positivi sul proprio apprendimento, sulle proprie competenze critiche, su una diffusa possibilità di apprezzare conoscenze e capacità dei colleghi, nonché proprie. Esiti analoghi emergono dall'analisi delle riflessioni che gli insegnanti producono quando invitati a confrontare il proprio lavoro con quello dei colleghi e ad individuare i principali risvolti dell'attività di peer-assessment rispetto al proprio percorso: mentre riflettono sulla stessa, infatti, i partecipanti realizzano che «valutando si impara», che «ripercorrere i percorsi messi in atto per la realizzazione dei prodotti e valutare la loro efficacia è stata un'importante occasione per consolidare le 
conoscenze apprese», e "di essere capace in poco tempo di lavorare con prodotti mai utilizzati prima, divertendomi a scoprire le potenzialità degli strumenti", confermando la possibilità che il feedback si trasformi in un inner generative process (Nicol, 2018), ovvero un learning tool per chi lo offre prima ancora che per chi lo riceve (Grion et al., 2017). Inevitabilmente, del resto, la riflessione si sposta dall'impatto dell'attività svolta su sé stessi alle possibili applicazioni con i propri studenti, rafforzando le potenzialità formative della pratica stessa: "Mi sono resa conto dell'importanza della valutazione, della sua complessità. della sua progettazione e difficoltà ma anche della sua immensa potenzialità. Mentre ci suggerisce quanto sappiamo e come sappiamo, deve nel frattempo condurre ad ampliare comprensione e acquisizione di conoscenza".

\section{Conclusioni}

Il presente contributo ha inteso indagare la competenza valutativa di insegnanti in servizio coinvolti in un'attività di peer-assessment $\mathrm{e}$ le loro percezioni relativamente al valore della pratica in sé.

$\mathrm{Nel}$ complesso, le analisi mostrano come l'attività di peer-assessment così come proposta abbia stimolato, da un lato, un potenziamento di competenze professionalizzanti, quali pensiero critico (Lynch, McNamara e Seery, 2012) e metacognizione (Vickerman, 2009; Wen e Tsai, 2006), dall'altro, la motivazione ad apprendere (Topping, 2005), in definitiva, rafforzando il sentimento di appartenenza a una comunità di pratiche.

Lo studio conferma, quindi, le ricerche precedenti sul ruolo del peer-assessment come strategia che potenzia lo sviluppo di abilità metariflessive e professionalizzanti, anche nell'ambito della formazione di insegnanti in servizio (Foschi e Cecchinato, 2019) e non soltanto nell'ambito dell'approccio metodologico proposto (Lynch, McNamara e Seery, 2012; Sluijsmans et al., 2002; Poon et al., 2009). Di converso, è la configurazione specifica della pratica ad averla resa un utile learning tool: fasi successive di training e modellamento, trasparenza dei criteri di valutazione, esposizioni ripetute all'uso della rubric, riflessioni condivise e, in generale, un approccio positivo al feedback reciproco hanno, nel complesso, reso efficace e sostenibile l'esperienza di valutazione tra pari.

Ancora una volta (Carless et al., 2017), si sottolinea l'importanza di una solida progettazione didattica come punto di partenza di percorsi diversificati e ricchi in cui i discenti partecipano ad attività autentiche, producono conoscenza e artefatti che in seguito migliorano iterativamente e reciprocamente, sostenuti da momenti di riflessione guidata che sola riconduce il senso della pratica specifica alla propria professionalità più ampia (Sansone et al, 2019; Sansone e 
Ritella, 2020). Sperimentare il peer-assessment in un contesto sociale 'simulato' promuove, infatti, una conoscenza approfondita della pratica e stimola un ripensamento delle proprie strategie valutative, portando gli insegnanti a riflettere sui vantaggi e sulle possibili problematicità della loro adozione all'interno delle proprie classi (Cheng et al., 2010; Yilmaz, 2017). Il ripensamento metacognitivo delle proprie pratiche di valutazione, del resto, è un utile viatico per lo sviluppo di una nuova cultura valutativa, sia nei docenti, sia negli studenti, con l'obiettivo di costruire competenze professionalizzanti e di cittadinanza contraddistinte da libertà, responsabilità e eticità (Ibarra-Sáiz et al., 2020; Rivoltella, 2020).

\section{Riferimenti bibliografici}

Black P., Harrison C., Lee C., Marshall B., and Wiliam D. (2003). Assessment for Learning: Putting it into practice. Bickingham: Open University press.

Carless D., and Boud D. (2018). The development of student feedback literacy: enabling uptake of feedback. Assessment \& Evaluation in Higher Education, 43(8): 1315-1325. DOI: 10.1080/02602938.2018.1463354.

Carless D., Bridges S., Chan C., and Glofcheski R. (2017). Scaling up assessment for learning in higher education. Singapore: Springer.

Cesareni D., and Sansone N. (2019). Il peer-feedback collaborativo per il miglioramento continuo dei prodotti. Italian Journal of Educational Research, 139-156. «testo disponibile al sito: https://ojs.pensamultimedia.it/index.php/sird/article/view/3274» 20/08/2021.

Cheng M. M. H., Cheng A. Y. N., and Tang S. Y. F. (2010). Closing the gap between the theory and practice of teaching: Implications for teacher education programmes in Hong Kong. Journal of Education for Teaching, 36(1): 91-104. DOI: $10.1080 / 02607470903462222$

Foschi L. C., and Cecchinato G. (2019). Validity and reliability of peer-grading in inservice teacher training. Italian Journal Of Educational Research: 177-194. «testo disponibile al sito: https://ojs.pensamultimedia.it/index.php/sird/article/view/3276» 20/08/2021

Foschi L.C., Cecchinato G., and Say F. (2019). Quis iudicabit ipsos iudices? Analisi dello sviluppo di competenze in un percorso di formazione per insegnanti tramite la valutazione tra pari e l'autovalutazione. Italian Journal of Educational Technology, 27(1): 49-64. DOI: 10.17471/2499-4324/1019.

Gielen S, Dochy F. and Dierick S. (2003). Evaluation the Consequential Validity of New Modes of Assessment: The Influence of Assessment on Learning, Including Pre-, Post- and True Assessment Effects. In Segers M., Dochy F. and Cascallar E., a cura di, Optimising New Modes of Assessment: In Search of Qualities and Standards. Dordrech: Springer. DOI: 10.1007/0-306-48125-1_3.

Giovannini L., Loiodice I., Lucisano P., Portera A., a cura di (2017), Strategie orientative e transizione università-lavoro. Roma: Armando. 
Grion V., and Tino C. (2018). Verso una "valutazione sostenibile" all'università: percezioni di efficacia dei processi di dare e ricevere feedback fra pari. Lifelong Lifewide Learning, 14(31): 38-55. DOI: 10.19241/11l.v14i31.104.

Grion V., Serbati A., Tino C., and Nicol D. (2017). Ripensare la teoria della valutazione e dell'apprendimento all'università: un modello per implementare pratiche di peer review. Giornale Italiano della Ricerca Educativa, 19: 209-226. «testo disponibile al sito: https://ojs.pensamultimedia.it/index.php/sird/article/view/2554» 20/08/2021.

Hanrahan S. J. and Isaacs G. (2001) Assessing Self- and Peer-assessment: The students' views. Higher Education Research \& Development, 20(1): 53-70. DOI: $10.1080 / 07294360123776$.

Hattie J., and Timperley H. (2007). The power of feedback. Review of Educational Research, 77(1): 81-112. DOI: 10.3102/003465430298487.

Ibarra-Sáiz M. S., Rodríguez-Gómez G., Boud D., Rotsaert T., Brown S., Salinas-Salazar M.L., and Rodríguez- Gómez H. M. (2020). El futuro de la evaluación en la educación superior. RELIEVE, 26(1): 1-6. DOI:10.7203/relieve.26.1.17323.

Leahy S., Lyon C., Thompson M. and Wiliam D. (2005). Classroom assessment: Minute by minute, day by day. Educational Leadership, 63(3): 18-24. «testo disponibile al sito: https://www.rbteach.com/sites/default/files/classroomassessment-minute-by-minute-day-by-day.pdf» 20/08/2021.

Lipnevich A.A., and Smith J.K. (2009). Effects of differential feedback on students' examination performance. Journal of Experimental Psychology: Applied, 15(4): 319-333. DOI: $10.1037 / \mathrm{a} 0017841$.

Liu X., and Li L. (2014). Assessment training effects on student assessment skills and task performance in a technology-facilitated peer assessment. Assessment \& Evaluation in Higher Education, 39(3): 275-292. DOI: 10.1080/02602938.2013.823540.

Lynch R., McNamara P. M., and Seery N. (2012). Promoting deep learning in a teacher education programme through self- and peer-assessment and feedback. European Journal of Teacher Education, 35(2): 179-197. DOI: 10.1080/02619768.2011.643396.

Magnoler P., Notti A.M. and Perla L., a cura di (2017). La professionalità degli insegnanti. La ricerca e le pratiche. Lecce: PensaMultimedia.

Nicol D. (2018). Unlocking generative feedback through peer reviewing. In Grion V. and Serbati A., a cura di, Valutare l'apprendimento o valutare per l'apprendimento? Verso una cultura della valutazione sostenibile all'Università. Lecce: Pensa Multimedia.

O’Donovan B., Rust C. and Price M. (2016). A Scholarly Approach to Solving the Feedback Dilemma in Practice. Assessment and Evaluation in Higher Education, 41(6): 938-949. DOI: 10.1080/02602938.2015.1052774.

Orpen, C. (1982). Student Versus Lecturer Assessment of Learning: A Research Note. Higher education, 11: 567-572. DOI: 10.1007/BF00194421.

Panadero E., Alonso-Tapia J., and Huertas J. A. (2012). Rubrics and self-assessment scripts effects on self-regulation, learning and self-efficacy in secondary education. 
Learning and Individual Differences, 22(6): 806-813. DOI: 10.1016/j.lindif.2012.04.007.

Poon W., McNaught C., Lam P., and Kwan H. S. (2009). Improving assessment methods in university science education with negotiated self-and peer-assessment. Assessment in Education: Principles, Policy and Practice, 16(3): 331-346. DOI: 10.1080/09695940903319729.

Rivoltella P. C., (2020). Nuovi alfabeti. Educazione e culture nella società postmediale. Brescia: Scholè.

Rivoltella P. C., Rossi P.G., a cura di (2017). L'agire didattico. Manuale per l'insegnante. Brescia: La scuola.

Rushton C., Ramsey P. and Rada R. (1993). Peer assessment in a collaborative hypermedia environment: A case study. Journal of Computer-Based Instruction, 20: $75-80$.

Sadler D. R. (1989). Formative assessment and the design of instructional systems. Instructional science, 18(2):119-144. DOI: 10.1007/BF00117714.

Sansone N. (2020). E-tivity. In: Limone P., Toto G.A., Sansone N., a cura di, Didattica universitaria a distanza. Tra emergenze e futuro. Progedit, Bari (2020).

Sansone N., and Ritella G. (2020). Formazione insegnanti "aumentata": integrazione di metodologie e tecnologie al servizio di una didattica socio-costruttivista. QwertyOpen and Interdisciplinary Journal of Technology, Culture and Education, 15(1): 70-88. «testo disponibile al sito: https://www.ckbg.org/qwerty/index.php/qwerty/article/view/372» 20/08/2021.

Sansone N., Bortolotti I., and Buglass S. (2016). The trialogical learning approach in practices: Reflections from pedagogical cases. Qwerty-Open and Interdisciplinary Journal of Technology, Culture and Education, 11(2): 99-120. «testo disponibile al sito: https://www.ckbg.org/qwerty/index.php/qwerty/article/viewFile/240/232» 20/08/2021

Sansone N., Cesareni D., Bortolotti I., and Buglass S. (2019). Teaching technologymediated collaborative learning for trainee teachers. Technology, Pedagogy and Education, 28(3): 381-394. DOI: 10.1080/1475939X.2019.1623070.

Sansone N., Cesareni D., Ligorio M. B., Bortolotti I., \& Buglass S. L. (2020). Developing knowledge work skills in a university course. Research Papers in Education, 35(1): 23-42. DOI: 10.1080/02671522.2019.1677754.

Schartel S. A. (2012). Giving feedback - An integral part of education. Best practice \& research Clinical anaesthesiology, 26(1): 77-87. DOI: 10.1016/j.bpa.2012.02.003.

Sibilio M. and Aiello P., a cura di (2018). Lo sviluppo professionale dei docenti. Ragionare di agentività per una scuola inclusiva. Napoli: EdiSES.

Sluijsmans D. M. A., Brand-Gruwel S., and van Merriënboer J. J. G. (2002). Peer assessment training in teacher education: Effects on performance and perceptions. Assessment and Evaluation in Higher Education, 27(5): 443-454. DOI: 10.1080/0260293022000009311.

Strijbos J. V., and Sluijsmans D. (2010). Unraveling peer assessment: methodological, functional and conceptual developments. Learning and Instruction, 20: 265-269. DOI: 10.1016/j.learninstruc.2009.08.002. 
Topping K. J. (1998). Peer Assessment Between Students in Colleges and Universities. Review of Educational Research, 68(3): 249-276. DOI: $10.3102 / 00346543068003249$.

Topping K. J. (2005). Trends in peer learning. Educational Psychology, 25(6): 631645. DOI: 10.1080/01443410500345172.

Topping K. J. (2017). Peer Assessment: Learning by Judging and Discussing the Work of Other Learners. Interdisciplinary Education and Psychology, 1(1): 1-17. DOI: 10.31532/InterdiscipEducPsychol.1.1.007.

Topping K. J. (2021). Peer Assessment: Channels of Operation. Education Sciences, 11(3): 91. DOI: 10.3390/educsci11030091.

Vannini I. (2019). Valutare per apprendere e progettare. In: Nigris E., Balconi B. and Zecca L., a cura di, Dalla progettazione alla valutazione didattica. Progettare, documentare e monitorare. Milano-Torino: Pearson Italia.

Vickerman P. (2009). Student perspectives on formative peer assessment: An attempt to deepen learning?. Assessment and Evaluation in Higher Education, 34(2): 221230. DOI: $10.1080 / 02602930801955986$.

Wen M. L. and Tsai C. (2006). University Students' Perceptions of and Attitudes Toward (Online) Peer Assessment. Higher Education, 51: 27-44. DOI: 10.1007/s10734-004-6375-8.

Wiliam D., Lee, C., Harrison C. and Black P. (2004). Teachers Developing Assessment for Learning: Impact on Student Achievement. Assessment in Education, 11(1): 4965. DOI: 10.1080/0969594042000208994.

Yilmaz F. M. (2017). Reliability of scores obtained from self-, peer-, and teacherassessments on teaching materials prepared by teacher candidates. Educational Sciences: Theory and Practice, 17(2): 395-409. DOI: 10.12738/estp.2017.2.0098. 